\title{
Stable Model Theory for Extended RDF Ontologies ${ }^{\star}$
}

\author{
Anastasia Analyti ${ }^{1}$, Grigoris Antoniou ${ }^{1,2}$, \\ Carlos Viegas Damásio ${ }^{3}$, and Gerd Wagner ${ }^{4}$ \\ ${ }^{1}$ Institute of Computer Science, FORTH-ICS, Greece \\ \{analyti, antoniou\}@ics.forth.gr \\ ${ }^{2}$ Department of Computer Science, University of Crete, Greece \\ ${ }^{3}$ Centro de Inteligência Artificial, Universidade Nova de Lisboa, Portugal \\ cd@di.fct.unl.pt \\ ${ }^{4}$ Inst. of Informatics, Brandenburg Univ. of Technology at Cottbus, Germany \\ G.Wagner@tu-cottbus .de
}

\begin{abstract}
Ontologies and automated reasoning are the building blocks of the Semantic Web initiative. Derivation rules can be included in an ontology to define derived concepts based on base concepts. For example, rules allow to define the extension of a class or property based on a complex relation between the extensions of the same or other classes and properties. On the other hand, the inclusion of negative information both in the form of negation-as-failure and explicit negative information is also needed to enable various forms of reasoning. In this paper, we extend RDF graphs with weak and strong negation, as well as derivation rules. The ERDF stable model semantics of the extended framework (Extended $R D F$ ) is defined, extending $\mathrm{RDF}(\mathrm{S})$ semantics. A distinctive feature of our theory, which is based on partial logic, is that both truth and falsity extensions of properties and classes are considered, allowing for truth value gaps. Our framework supports both closed-world and open-world reasoning through the explicit representation of the particular closed-world assumptions and the ERDF ontological categories of total properties and total classes.
\end{abstract}

\section{Introduction}

The idea of the Semantic Web is to describe the meaning of web data in a way suitable for automated reasoning. This means that descriptive data (meta-data) in machine readable form are to be stored on the web and used for reasoning. Due to its distributed and world-wide nature, the Web creates new problems for knowledge representation research. In [2], the following fundamental theoretical problems have been identified: negation and contradictions, open-world versus closed-world assumptions, and rule systems for the Semantic Web. For the time being, the first two issues have been circumvented by discarding the facilities to

\footnotetext{
* This research has been partially funded by European Commission and by the Swiss Federal Office for Education and Science within the 6th Framework Programme project REWERSE number 506779 (www.rewerse.net).
} 
introduce them, namely negation and closed-world assumptions. Though the web ontology language OWL [13, which is based on description logic (DL), includes a form of classical negation through class complements, this form is limited. This is because, to achieve decidability, classes are formed based on specific class constructors and negation on properties is not considered. Rules constitute the next layer over the ontology languages of the Semantic Web and, in contrast to DL, allow arbitrary interaction of variables in the body of the rules. The widely recognized need of having rules in the Semantic Web [10 14 has restarted the discussion of the fundamentals of closed-world reasoning and the appropriate mechanisms to implement it in rule systems, such as the computational concept of negation-as-failure.

The $\mathrm{RDF}(\mathrm{S})$ recommendation [6] provides the basic constructs for defining web ontologies and a solid ground to discuss the above issues. $\mathrm{RDF}(\mathrm{S})$ is a special predicate logical language that is restricted to existentially quantified conjunctions of atomic formulas, involving binary predicates only. Thus, RDF(S) does not support negation and rules. In [18, it was argued that a database, as a knowledge representation system, needs two kinds of negation, namely weak negation $\sim$ (expressing negation-as-failure or not-truth) and strong negation $\neg$ (expressing explicit negative information or falsity) to be able to deal with partial information. In [19], this point was made for the Semantic Web as a framework for knowledge representation in general. In the present paper we make the same point for the Semantic Web language RDF and show how it can be extended to accommodate the two negations of partial logic [7], as well as derivation rules. We call the extended language Extended $R D F$ and denote it by ERDF. The model-theoretic semantics of ERDF, called ERDF stable model semantics, is developed based on partial logic [7.

In partial logic, relating strong and weak negation at the interpretation level allows to distinguish four categories of properties and classes. Partial properties are properties $p$ that may have truth-value gaps and truth-value clashes, that is $p(x, y)$ is possibly neither true nor false, or both true and false. Total properties are properties $p$ that satisfy totalness, that is $p(x, y)$ is true or false (but possibly both). Coherent properties are properties $p$ that satisfy coherence, that is $p(x, y)$ cannot be both true and false. Classical properties are total and coherent properties. For classical properties $p$, the classical logic law applies: $p(x, y)$ is either true or false. Partial, total, coherent, and classical classes $c$ are defined similarly, by replacing $p(x, y)$ by $r d f: \operatorname{type}(x, c)$.

Partial logic allows also to distinguish between properties (similarly, classes) that are completely represented in a knowledge base and those that are not. The classification if a property is completely represented or not is up to the owner of the knowledge base: the owner must know for which properties there is complete information and for which there is not. Clearly, in the case of a completely represented (closed) predicate $p$, negation-as-failure implies falsity, and the underlying completeness assumption is also called Closed-World Assumption $(C W A)$. A CWA for $p$ is represented in our framework through the inclusion of the derivation rule $\neg p(? x, ? y) \leftarrow \sim p(? x, ? y)$ (for a closed class $c$, the correspond- 
ing CWA is $\neg r d f: \operatorname{type}(? x, c) \leftarrow \sim r d f$ :type $(? x, c))$. In the case of an incompletely represented (open) predicate $p$, negation-as-failure is not applicable and explicit negative information has to be supplied along with ordinary (positive) information. In particular, the inclusion of the derivation rule $\neg p(? x, ? y) \leftarrow \sim p(? x, ? y)$ will not affect the semantics of $p$. Unfortunately, neither classical logic nor Prolog supports this distinction between "closed" and "open" predicates. Classical logic supports only open-world reasoning. On the contrary, Prolog supports only closed-world reasoning, as negation-as-failure is the only negation mechanism supported. For arguments in favor of the combination of closed and open world reasoning in the same framework, see [1].

Specifically, in this paper:

1. We extend RDF graphs to ERDF graphs with the inclusion of strong negation, and then to ERDF ontologies (or ERDF knowledge bases) with the inclusion of general derivation rules. ERDF graphs allow to express existential positive and negative information, whereas general derivation rules allow inferences based on formulas built using the connectives $\sim, \neg, \supset, \wedge, \vee$ and the quantifiers $\forall, \exists$.

2. We extend the vocabulary of $\operatorname{RDF}(\mathrm{S})$ with the terms erdf:TotalProperty and erdf:TotalClass, representing metaclasses of total properties and total classes, on which the open-world assumption applies.

3. We extend RDFS interpretations to ERDF interpretations including both truth and falsity extensions for properties and classes. Then, we define coherent ERDF interpretations by imposing coherence on all properties. In the developed model-theoretic semantics of ERDF, we consider only coherent ERDF interpretations. Thus, total properties and classes become synonymous to classical properties and classes.

4. We extend RDF graphs to ERDF formulas that are built from positive triples using the connectives $\sim, \neg, \supset, \wedge, \vee$ and the quantifiers $\forall, \exists$. Then, we define ERDF entailment between two ERDF formulas, extending RDFS entailment between RDF graphs.

5. We define the ERDF models, Herbrand interpretations, minimal Herbrand models, and stable models of ERDF ontologies. We show that stable model entailment on ERDF ontologies extends RDFS entailment on RDF graphs.

6. We show that if all properties are total, classical (boolean) Herbrand model reasoning and stable model reasoning coincide. In this case, we make an open-world assumption for all properties and classes.

The rest of the paper is organized as follows: In Section 2 , we extend RDF graphs to ERDF graphs and ERDF formulas. Section 3 defines ERDF interpretations and ERDF entailment. We show that ERDF entailment extends RDFS entailment. In Section 4, we define ERDF ontologies and the Herbrand models of an ERDF ontology. In Section 5, we define the stable models of an ERDF ontology and show that stable model entailment extends RDFS entailment. Section 6 reviews related work and Section 7 concludes the paper. 


\section{Extending RDF Graphs with Negative Information}

In this section, we extend RDF graphs to ERDF graphs, by adding strong negation. Moreover, we extend RDF graphs to ERDF formulas, which are built from positive ERDF triples, the connectives $\sim, \neg, \supset, \wedge, \vee$, and the quantifiers $\forall, \exists$.

According to RDF concepts 12 6, URI references are used for naming web resources. A URI reference consists of two parts: a namespace URI $n s$ and a local name $l n$, and is denoted by $n s: l n$. A plain literal is a string " $s$ ", where $s$ is a sequence of Unicode characters, or a pair of a string " $s$ " and a language tag $t$, denoted by " $s$ " @ $t$. A typed literal is a pair of a string " $s$ " and a datatype URI reference $d$, denoted by " $s " \wedge$. A (Web) vocabulary $V$ is a set of URI references and/or literals (plain or typed). We denote the set of all URI references by URI, the set of all plain literals by $\mathcal{P} \mathcal{L}$, the set of all typed literals by $\mathcal{T} \mathcal{L}$, and the set of all literals by $\mathcal{L} \mathcal{I} \mathcal{T}$.

In our formalization, we consider a set Var of variable symbols, such that the sets Var, URI, $\mathcal{L I} \mathcal{T}$ are pairwise disjoint. In the main text, variable symbols are explicitly indicated, while in our examples, variable symbols are prefixed by ?.

Below we extend the notion of RDF triple to allow for both positive and negative information.

Definition 1 (ERDF triple). Let $V$ be a vocabulary. A positive ERDF triple over $V$ (also called ERDF sentence atom) is an expression of the form $p(s, o)$, where $s, o \in V \cup \operatorname{Var}$ are called subject and object, respectively, and $p \in V \cap U R I$ is called predicate or property.

A negative ERDF triple over $V$ is the strong negation $\neg p(s, o)$ of a positive ERDF triple $p(s, o)$ over $V$.

An ERDF triple over $V$ (also called ERDF sentence literal) is a positive or negative ERDF triple over $V$.

For example, ex:likes(ex:Gerd, ex:Riesling) is a positive ERDF triple, and $\neg$ ex: likes(ex:Carlos, ex:Riesling) is a negative ERDF triple. Note that an RDF triple is a positive ERDF triple with the constraint that the subject of the triple is not a literal. For example, ex:nameOf("Grigoris", ex:Grigoris) is a valid ERDF triple but not a valid RDF triple. Our choice of allowing literals appearing in the subject position is based on our intuition that this case can naturally appear in knowledge representation (as in the previous example). Moreover, note that a variable in the object position of an ERDF triple in the body of a rule, can appear in the subject position of the ERDF triple in the head of the rule. Since variables can be instantiated by a literal, a literal can naturally appear in the subject position of the derived ERDF triple.

Definition 2 (ERDF formula). Let $V$ be a vocabulary. We consider the logical factors $\{\sim, \neg, \wedge, \vee, \supset, \exists, \forall\}$, where $\neg, \sim$, and $\supset$ are called strong negation, weak negation, and material implication respectively. We denote by $L(V)$ the smallest set that contains the positive ERDF triples over $V$ and is closed with respect to the following conditions: if $F, G \in L(V)$ then $\{\sim F, \neg F, F \wedge G, F \vee G$, $F \supset G, \exists x F, \forall x F\} \subseteq L(V)$, where $x \in \operatorname{Var}$. An ERDF formula over $V$ is an 
element of $L(V)$. We denote the set of variables appearing in $F$ by $\operatorname{Var}(F)$, and the set of free variables 1 appearing in $F$ by $F \operatorname{Var}(F)$.

For example, let $F=\forall ? x \exists ? y$ (rdf:type(?x, ex:Person) $\supset$ ex:hasFather $(? x, ? y))$ $\wedge$ rdf:type(?z,ex:Person). Then, $F$ is an ERDF formula over the vocabulary $V=\{r d f:$ type, ex:Person, ex:hasFather $\}$ with $\operatorname{Var}(F)=\{? x, ? y, ? z\}$ and $F \operatorname{Var}(F)=\{? z\}$.

We will denote the sublanguages of $L(V)$ formed by means of a subset $S$ of the logical factors, by $L(V \mid S)$. For example, $L(V \mid\{\neg\})$ denotes the set of (positive and negative) ERDF triples over $V$.

Definition 3 (ERDF graph). An ERDF graph $G$ is a set of ERDF triples over some vocabulary $V$. We denote the variables appearing in $G$ by $\operatorname{Var}(G)$, and the set of URI references and literals appearing in $G$ by $V_{G}$.

Intuitively, an ERDF graph $G$ represents an existentially quantified conjunction of $E R D F$ triples. Specifically, let $G=\left\{t r_{1}, \ldots, t r_{n}\right\}$ be an $E R D F$ graph, and let $\operatorname{Var}(G)=\left\{x_{1}, \ldots x_{k}\right\}$. Then, $G$ represents the formula $\exists x_{1}, \ldots x_{k} t r_{1} \wedge \ldots \wedge t r_{n}$. Following the RDF terminology [12, the variables of an ERDF graph are called blank nodes, and intuitively denote anonymous web resources.

Note that as an RDF graph is a set of RDF triples [126], an RDF graph is also an ERDF graph.

\section{$3 \quad$ ERDF Interpretations}

In this section, we extend $\operatorname{RDF}(\mathrm{S})$ semantics by allowing for partial properties and classes. In particular, we define ERDF interpretations and satisfaction of an ERDF formula. For simplicity, we disregard RDF $(\mathrm{S})$ containers, collections, and reification, as no special semantic conditions are imposed on these, and thus can be included by a straightforward extension.

Below we define a partial interpretation as an extension of a simple interpretation [6], where each property is associated not only with a truth extension but also with a falsity extension allowing for partial properties.

Definition 4 (Partial interpretation). A partial interpretation I of a vocabulary $V$ consists of:

- A non-empty set of resources $\operatorname{Res}_{I}$, called the domain or universe of $I$.

- A set of properties $\operatorname{Prop}_{I}$.

- A vocabulary interpretation mapping $I_{V}: V \cap U R I \rightarrow \operatorname{Res}_{I} \cup \operatorname{Prop}_{I}$.

- A property-truth extension mapping $P T_{I}: \operatorname{Prop}_{I} \rightarrow \mathcal{P}\left(\operatorname{Res}_{I} \times \operatorname{Res}_{I}\right)$.

- A property-falsity extension mapping $P F_{I}: \operatorname{Prop}_{I} \rightarrow \mathcal{P}\left(\operatorname{Res}_{I} \times \operatorname{Res}_{I}\right)$.

- A mapping $I L_{I}: V \cap \mathcal{T} \mathcal{L} \rightarrow \operatorname{Res}_{I}$.

- A set of literal values $L V_{I} \subseteq R e s_{I}$, which contains $V \cap \mathcal{P} \mathcal{L}$.

We define the mapping: $I: V \rightarrow \operatorname{Res}_{I} \cup \operatorname{Prop}_{I}$ such that:

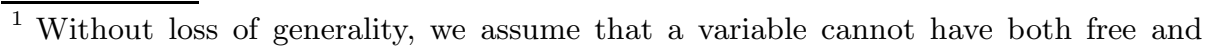
bound occurrences in $F$, and more than one bound occurrence. 
$-I(x)=I_{V}(x), \forall x \in V \cap U R I$.

$-I(x)=x, \forall x \in V \cap \mathcal{P} \mathcal{L}$.

- $I(x)=I L_{I}(x), \forall x \in V \cap \mathcal{T} \mathcal{L}$.

Definition 5 (Satisfaction of an ERDF formula w.r.t. a partial interpretation and a valuation). Let $F, G$ be $E R D F$ formulas and let $I$ be a partial interpretation of a vocabulary $V$. Let $v$ be a mapping $v: \operatorname{Var}(F) \rightarrow \operatorname{Res}_{I}$ (called valuation). If $x \in \operatorname{Var}(F)$, we define $[I+v](x)=v(x)$. If $x \in V$, we define $[I+v](x)=I(x)$.

- If $F=p(s, o)$ then $I, v \models F$ iff $p \in V \cap U R I, s, o \in V \cup \operatorname{Var}, I(p) \in \operatorname{Prop}_{I}$, and $\langle[I+v](s),[I+v](o)\rangle \in P T_{I}(I(p))$.

- If $F=\neg p(s, o)$ then $I, v \models F$ iff $p \in V \cap U R I, s, o \in V \cup V a r, I(p) \in$ Prop $_{I}$, and $\langle[I+v](s),[I+v](o)\rangle \in P F_{I}(I(p))$.

- If $F=\sim G$ then $I, v \mid=F$ iff all URIs and literals appearing in $G$ belong to $V$, and $I, v \not \forall G$.

- If $F=F_{1} \wedge F_{2}$ then $I, v \models F$ iff $I, v \models F_{1}$ and $I, v \models F_{2}$.

- If $F=F_{1} \vee F_{2}$ then $I, v \models F$ iff $I, v \models F_{1}$ or $I, v \models F_{2}$.

- If $F=F_{1} \supset F_{2}$ then $I, v \models F$ iff $I, v \models \sim F_{1} \vee F_{2}$.

- If $F=\exists x G$ then $I, v \models F$ iff there exists mapping $u: \operatorname{Var}(G) \rightarrow \operatorname{Res}_{I}$ such that $u(y)=v(y), \forall y \in \operatorname{Var}(G)-\{x\}$, and $I, u \models G$.

- If $F=\forall x G$ then $I, v \models F$ iff for all mappings $u: \operatorname{Var}(G) \rightarrow \operatorname{Res}_{I}$ such that $u(y)=v(y), \forall y \in \operatorname{Var}(G)-\{x\}$, it holds $I, u=G$.

- All other cases of ERDF formulas are treated by the following DeMorgan-style rewrite rules expressing the falsification of compound ERDF formulas:

$\neg(F \wedge G) \rightarrow \neg F \vee \neg G, \neg(F \vee G) \rightarrow \neg F \wedge \neg G, \neg \neg F \rightarrow F, \neg \sim F \rightarrow F$, $\neg \exists x F \rightarrow \forall x \neg F, \quad \neg \forall x F \rightarrow \exists x \neg F, \neg(F \supset G) \rightarrow F \wedge \neg G$.

Definition 6 (Satisfaction of an ERDF formula w.r.t. a partial interpretation). Let $F$ be an $E R D F$ formula and let $I$ be a partial interpretation of a vocabulary $V$. We say that $I$ satisfies $F$, denoted by $I \models F$, iff for every mapping $v: \operatorname{Var}(F) \rightarrow \operatorname{Res}_{I}$, it holds $I, v \models F$.

Note that as an ERDF graph represents an existentially quantified conjunction of ERDF triples, the above definition applies also to ERDF graphs. Specifically, let $G$ be an ERDF graph representing the formula $F=\exists x_{1}, \ldots x_{k} t r_{1} \wedge \ldots \wedge t r_{n}$. We say that a partial interpretation $I$ satisfies the ERDF graph $G(I \models G)$ iff $I \models F$.

We are now ready to define an ERDF interpretation over a vocabulary $V$ as an extension of an RDFS interpretation [6], where each property and class is associated not only with a truth extension but also with a falsity extension, allowing for both partial properties and partial classes. Additionally, an ERDF interpretation gives special semantics to terms from the ERDF vocabulary.

The vocabulary of RDF, $\mathcal{V}_{R D F}$, and the vocabulary of RDFS, $\mathcal{V}_{R D F S}$, are defined in [6]. The vocabulary of ERDF, $\mathcal{V}_{E R D F}$, is a set of $U R I$ references in the erdf: namespace. Specifically, the set of ERDF predefined classes is $\mathcal{C}_{E R D F}=$ $\{$ erdf:TotalClass, erdf:TotalProperty $\}$. We define $\mathcal{V}_{E R D F}=\mathcal{C}_{E R D F}$. Intuitively, instances of the metaclass erdf:TotalClass are classes $c$ that satisfy totalness, meaning that each resource belongs to the truth or falsity extension of 
c. Similarly, instances of the metaclass erdf:TotalProperty are properties $p$ that satisfy totalness, meaning that each pair of resources belongs to the truth or falsity extension of $p$.

Definition 7 (ERDF interpretation). An ERDF interpretation $I$ of a vocabulary $V$ is a partial interpretation of $V \cup \mathcal{V}_{R D F} \cup \mathcal{V}_{R D F S} \cup \mathcal{V}_{E R D F}$, extended by the new ontological categories $C l s_{I} \subseteq R e s_{I}$ for classes, $T C l s_{I} \subseteq C l s_{I}$ for total classes, and $\operatorname{TProp}_{I} \subseteq \operatorname{Prop}_{I}$ for total properties, as well as the classtruth extension mapping $C T_{I}: C l s_{I} \rightarrow \mathcal{P}\left(R e s_{I}\right)$, and the class-falsity extension mapping $C F_{I}: C l s_{I} \rightarrow \mathcal{P}\left(\operatorname{Res}_{I}\right)$, such that:

1. $x \in C T_{I}(y)$ iff $\langle x, y\rangle \in P T_{I}(I(r d f: t y p e))$, and $x \in C F_{I}(y)$ iff $\langle x, y\rangle \in P F_{I}(I(r d f:$ type $))$.

2. The ontological categories are defined as follows:

$$
\begin{aligned}
& \left.\operatorname{Prop}_{I}=C T_{I}(I(r d f: \text { Property })) \quad C l s_{I}=C T_{I}(I \text { (rdfs:Class })\right) \\
& \left.\operatorname{Res}_{I}=C T_{I}(I(\text { rdfs:Resource })) \quad L V_{I}=C T_{I}(I \text { (rdfs:Literal })\right) \\
& T C l s_{I}=C T_{I}(I(\text { erdf:TotalClass })) \text { TProp }_{I}=C T_{I}(I(\text { erdf:TotalProperty })) .
\end{aligned}
$$

3. if $\langle x, y\rangle \in P T_{I}(I$ (rdfs:domain $\left.)\right)$ and $\langle z, w\rangle \in P T_{I}(x)$ then $z \in C T_{I}(y)$.

4. If $\langle x, y\rangle \in P T_{I}(I(r d f s:$ range $))$ and $\langle z, w\rangle \in P T_{I}(x)$ then $w \in C T_{I}(y)$.

5. If $x \in C l s_{I}$ then $\langle x, I$ (rdfs:Resource $\left.)\right\rangle \in P T_{I}(I$ (rdfs:subclassOf $\left.)\right)$.

6. If $\langle x, y\rangle \in P T_{I}(I(r d f s: s u b C l a s s O f))$ then $x, y \in C l s_{I}, C T_{I}(x) \subseteq C T_{I}(y)$, and $C F_{I}(y) \subseteq C F_{I}(x)$.

7. $P T_{I}(I(r d f s: s u b C l a s s O f))$ is a reflexive and transitive relation on $C l_{s_{I}}$.

8. If $\langle x, y\rangle \in P T_{I}(I(r d f s: s u b P r o p e r t y O f))$ then $x, y \in \operatorname{Prop}_{I}, P T_{I}(x) \subseteq P T_{I}(y)$, and $P F_{I}(y) \subseteq P F_{I}(x)$.

9. $P T_{I}(I(r d f s: s u b P r o p e r t y O f))$ is a reflexive and transitive relation on Prop $_{I}$.

10. If $x \in C T_{I}(I(r d f s:$ Datatype $))$ then $\langle x, I(r d f s:$ Literal $)\rangle \in P T_{I}(I(r d f s: s u b C l a s s O f))$.

11. If $x \in T C l s_{I}$ then $C T_{I}(x) \cup C F_{I}(x)=R e s_{I}$.

12. If $x \in$ TProp $_{I}$ then $P T_{I}(x) \cup P F_{I}(x)=\operatorname{Res}_{I} \times \operatorname{Res}_{I}$.

13. If " $s " \wedge r d f: X M L$ Literal $\in V$ and $s$ is a well-typed XML literal string, then $I L_{I}(" s " \wedge \wedge d f: X M L$ Literal) is the XML value of $s$,

$I L_{I}(" s " \wedge \wedge d f: X M L$ Literal $) \in L V_{I}$, and

$I L_{I}(" s " \wedge \wedge d f: X M L L i t e r a l) \in C T_{I}(I(r d f: X M L$ Literal $))$.

14. If " $s " \wedge r d f: X M L L i t e r a l \in V$ and $s$ is an ill-typed XML literal string then

$I L_{I}(" s " \wedge$ rdf:XMLLiteral $) \in \operatorname{Res}_{I}-L V_{I}$, and

$I L_{I}(" s " \wedge r d f: X M L$ Literal $) \in C F_{I}(I(r d f s:$ Literal $))$.

15. I satisfies the RDF and RDFS axiomatic triples [6], as well as the ERDF axiomatic triples:

rdf s:subClassOf (erdf:TotalClass, rdf s:Class).

rdf s:subClassOf (erdf:TotalProperty, rdf:Property).

Note that the semantic conditions of ERDF interpretations may impose constraints to both the truth and falsity extensions of properties and classes.

Definition 8 (Coherent ERDF interpretation). An ERDF interpretation $I$ of a vocabulary $V$ is coherent iff for all $x \in \operatorname{Prop}_{I}, P T_{I}(x) \cap P F_{I}(x)=\emptyset$.

Coherent ERDF interpretations enforce the constraint that a pair of resources cannot belong to both the truth and falsity extensions of a property. Since $r d f$ :type is a property, this constraint also implies that a resource cannot belong to both the truth and falsity extensions of a class. 
In the rest of the document, we consider only coherent ERDF interpretations. This means that referring to an "ERDF interpretation", we implicitly mean a "coherent" one.

According to RDFS semantics, the only source of RDFS-inconsistency is the appearance of an ill-typed XML literal in the RDF graph (possibly causing an $X M L$ clash, for details see [6]). An ERDF graph can be ERDF-inconsistent2, not only due to the appearance of an ill-typed XML literal in the ERDF graph, but also due to the additional semantic condition for coherent ERDF interpretations.

For example, let $p, q, s, o \in U R I$ and let $G=\{p(s, o)$, rdf $s: s u b P r o p e r t y O f(p$, $q), \neg q(s, o)\}$. Then, $G$ is ERDF-inconsistent, since there is no (coherent) ERDF interpretation that satisfies $G$.

The following proposition shows that for total properties and total classes of (coherent) ERDF interpretations, weak negation and strong negation coincide (boolean truth values).

Proposition 1. Let $I$ be an ERDF interpretation of a vocabulary $V$ and let $V^{\prime}=V \cup \mathcal{V}_{R D F} \cup \mathcal{V}_{R D F S} \cup \mathcal{V}_{E R D F}$. Then,

1. For all $p, s, o \in V^{\prime}$, such that $I(p) \in T \operatorname{Prop}_{I}$, it holds:

$I \models \sim p(s, o)$ iff $I \models \neg p(s, o)$ (equivalently, $I \models p(s, o) \vee \neg p(s, o)$ ).

2. For all $x, c \in V^{\prime}$ such that $I(c) \in T C l s_{I}$, it holds:

$I \models \sim r d f: \operatorname{type}(x, c)$ iff $I \models \neg r d f: \operatorname{type}(x, c)$

(equivalently, $I \models r d f: \operatorname{type}(x, c) \vee \neg r d f: \operatorname{type}(x, c))$.

Definition 9 (Classical ERDF interpretation). A (coherent) ERDF interpretation $I$ of a vocabulary $V$ is classical iff for all $x \in \operatorname{Prop}_{I}, \quad \operatorname{PT}_{I}(x) \cup$ $P F_{I}(x)=\operatorname{Res}_{I} \times \operatorname{Res}_{I}$.

A classical ERDF interpretation is close to an interpretation of classical logic, since for every formula $F$, weak and strong negation coincide.

Proposition 2. Let $I$ be an ERDF interpretation of a vocabulary $V$ and let $V^{\prime}=V \cup \mathcal{V}_{R D F} \cup \mathcal{V}_{R D F S} \cup \mathcal{V}_{E R D F}$. Then,

1. If $T \operatorname{Prop}_{I}=\operatorname{Prop}_{I}$ then $I$ is a classical ERDF interpretation.

2. If $I$ is a classical ERDF interpretation and $F$ is an ERDF formula over $V^{\prime}$ such that $I(p) \in \operatorname{Prop}_{I}$, for every property $p$ in $F$, then it holds: $I \models \sim F$ iff $I \models \neg F$ (equivalently, $I \models F \vee \neg F$ ).

The following definition defines ERDF entailment between two ERDF formulas.

Definition 10 (ERDF Entailment). Let $F, F^{\prime}$ be ERDF formulas. We say that $F$ ERDF-entails $F^{\prime}\left(F \models E R D F \quad F^{\prime}\right)$ iff for every ERDF interpretation $I$, if $I \models F$ then $I \models F^{\prime}$.

For example, let $F=\forall ? x \exists ? y$ (rdf:type(?x,ex:Person) $\supset$ ex:hasFather $(? x, ? y))$ $\wedge$ rdf:type(ex:John, ex:Person), and let $F^{\prime}=\exists$ ?y ex:hasFather(ex:John,?y) $\wedge r d f:$ type (ex:hasFather, $r d f:$ Property). Then $F \models{ }^{E R D F} F^{\prime}$.

The following proposition shows that an RDF graph is RDFS satisfiable iff it is ERDF satisfiable.

\footnotetext{
${ }^{2}$ Meaning that there is no (coherent) ERDF interpretation that satisfies the ERDF graph.
} 
Proposition 3. Let $G$ be an RDF graph such that $V_{G} \cap \mathcal{V}_{E R D F}=\emptyset$. Then, there is an RDFS interpretation that satisfies $G$ iff there is an ERDF interpretation that satisfies $G$.

The following proposition shows that ERDF entailment extends RDFS entailment from RDF graphs to ERDF formulas.

Proposition 4. Let $G, G^{\prime}$ be RDF graphs such that $V_{G} \cap \mathcal{V}_{E R D F}=\emptyset$ and $V_{G^{\prime}} \cap \mathcal{V}_{E R D F}=\emptyset$. Then, $G=^{R D F S} G^{\prime}$ iff $G \models{ }^{E R D F} G^{\prime}$.

\section{ERDF Ontologies}

In this section, we define an ERDF ontology as a pair of an ERDF graph $G$ and a set $P$ of ERDF rules. ERDF rules should be considered as derivation rules that allow us to infer more ontological information based on the declarations in $G$. Moreover, we define the Herbrand interpretations and the Herbrand models of an ERDF ontology.

Definition 11 (ERDF rule, ERDF program). An ERDF rule $r$ over a vocabulary $V$ is an expression of the form: $G \leftarrow F$, where $F \in L(V)$ is called condition and $G \in L(V \mid\{\neg\})$ is called conclusion. We assume that no bound variable in $F$ appears free in $G$. We denote the set of variables and the set of free variables of $r$ by $\operatorname{Var}(r)$ and $F \operatorname{Var}(r) \sqrt{3}$, respectively. Additionally, we write $\operatorname{Cond}(r)=F$ and $\operatorname{Concl}(r)=G$.

An ERDF program $P$ is a set of ERDF rules over some vocabulary $V$. We denote the set of URI references and literals appearing in $P$ by $V_{P}$.

Definition 12 (ERDF ontology). An ERDF ontology (or knowledge base) is a pair $O=\langle G, P\rangle$, where $G$ is an ERDF graph and $P$ is an ERDF program.

The following definition defines the models of an ERDF ontology.

Definition 13 (Satisfaction of an ERDF rule and an ERDF ontology). Let $I$ be an ERDF interpretation of a vocabulary $V$.

- We say that $I$ satisfies an ERDF rule $r$, denoted by $I \models r$, iff it holds: If there is a mapping $v: \operatorname{Var}(r) \rightarrow \operatorname{Res}_{I}$ such that $I, v \models \operatorname{Cond}(r)$ then $I, v \models \operatorname{Concl}(r)$.

- We say that $I$ satisfies an ERDF ontology $O=\langle G, P\rangle$ (also, $I$ is a model of $O$ ), denoted by $I \models O$, iff $I \models G$ and $I \models r, \forall r \in P$.

Definition 14 (Skolemization of an ERDF graph). Let $G$ be an ERDF graph. The skolemization function of $G$ is an 1:1 mapping $s k_{G}: \operatorname{Var}(G) \rightarrow U R I$, where for each $x \in \operatorname{Var}(G), s k_{G}(x)$ is an artificial URI denoted by $G: x$. The set $s k_{G}(\operatorname{Var}(G))$ is called the Skolem vocabulary of $G$.

The skolemization of $G$, denoted by $s k(G)$, is the ground ERDF graph derived from $G$ after replacing each variable $x \in \operatorname{Var}(G)$ by $s k_{G}(x)$.

${ }^{3} F \operatorname{Var}(r)=F \operatorname{Var}(F) \cup F \operatorname{Var}(G)$. 
Intuitively, the Skolem vocabulary of $G$ (that is, $\left.s k_{G}(\operatorname{Var}(G))\right)$ contains artificial URIs giving "arbitrary" names to the anonymous entities whose existence was asserted by the use of blank nodes in $G$.

Proposition 5. Let $G$ be an ERDF graph and let $I$ be an ERDF interpretation. Then, $I \models s k(G)$ implies $I \models G$.

Definition 15 (Vocabulary of an ERDF ontology). Let $O=\langle G, P\rangle$ be an ERDF ontology. The vocabulary of $O$ is defined as $V_{O}=V_{s k(G)} \cup V_{P} \cup \mathcal{V}_{R D F} \cup$ $\mathcal{V}_{R D F S} \cup \mathcal{V}_{E R D F}$.

Let $O=\langle G, P\rangle$ be an ERDF ontology. We denote by $\operatorname{Re} s_{O}^{H}$ the union of $V_{O}$ and the set of XML values of the well-typed XML literals in $V_{O}$ minus the well-typed XML literals.

Definition 16 (Herbrand interpretation, Herbrand model of an ERDF ontology). Let $O=\langle G, P\rangle$ be an ERDF ontology and let $I$ be an ERDF interpretation of $V_{O} . I$ is a Herbrand interpretation of $O$ iff:

$-\operatorname{Res}_{I}=\operatorname{Res}_{O}^{H}$.

$-I_{V}(x)=x$, for all $x \in V_{O} \cap U R I$.

- $I L_{I}(x)=x$, if $x$ is a typed literal in $V_{O}$ other than a well-typed XML literal, and $I L_{I}(x)$ is the XML value of $x$, if $x$ is a well-typed XML literal in $V_{O}$.

We denote the set of Herbrand interpretations of $O$ by $\mathcal{I}^{H}(O)$.

A Herbrand interpretation $I$ of $O$ is a Herbrand model of $O$ iff $I \models\langle s k(G), P\rangle$. We denote the set of Herbrand models of $O$ by $\mathcal{M}^{H}(O)$.

Obviously, every Herbrand model of an ERDF ontology $O$ is a model of $O$.

\section{Minimal Herbrand Interpretations and Stable Models}

In the previous section, we defined the Herbrand models of an ERDF ontology $O$. However, not all Herbrand models of $O$ are desirable. In this section, we define the intended models of $O$, called stable models of $O$, based on minimal Herbrand interpretations. In particular, defining the stable models of $O$, only the minimal interpretations from a set of Herbrand interpretations that satisfy certain criteria are considered.

For example, let $p, s, o \in U R I$, let $G=\{p(s, o)\}$ and let $O=\langle G, \emptyset\rangle$, Then, there is a Herbrand model $I$ of $O$ such that $I \models p(o, s)$, whereas we want $\sim p(o, s)$ to be satisfied by all intended models of $O$, as $p$ is not a total property 4 and $p(o, s)$ cannot be derived from $O$ (negation-as-failure).

To define the minimal Herbrand interpretations of an ERDF ontology $O$, we need to define a partial ordering on the Herbrand interpretations of $O$.

Definition 17 (Herbrand interpretation ordering). Let $O=\langle G, P\rangle$ be an ERDF ontology. Let $I, J \in \mathcal{I}^{H}(O)$. We say that $J$ extends $I$, denoted by $I \leq J$ (or $J \geq I$ ), iff $\operatorname{Prop}_{I} \subseteq \operatorname{Prop}_{J}$, and for all $p \in \operatorname{Prop}_{I}$, it holds $P T_{I}(p) \subseteq P T_{J}(p)$ and $P F_{I}(p) \subseteq P F_{J}(p)$.

\footnotetext{
${ }^{4}$ On total properties and classes, the open-world assumption applies.
} 
The intuition behind Definition 17 is that by extending a Herbrand interpretation, we extend both the truth and falsity extension for all properties, and thus (since $r d f$ :type is a property), for all classes.

Definition 18 (Minimal Herbrand Interpretations). Let $O$ be an ERDF ontology and let $\mathcal{I} \subseteq \mathcal{I}^{H}(O)$. We define $\operatorname{minimal}(\mathcal{I})=\{I \in \mathcal{I} \mid \nexists J \in \mathcal{I}: J \neq I$ and $J \leq I\}$.

Let $I, J \in \mathcal{I}^{H}(O)$, we define $[I, J]_{O}=\left\{I^{\prime} \in \mathcal{I}^{H}(O), I \leq I^{\prime} \leq J\right\}$. Additionally, we define the minimal Herbrand models of $O$, as $\mathcal{M}^{\min }(O)=\operatorname{minimal}\left(\mathcal{M}^{H}(O)\right)$.

However minimal Herbrand models do not give the intended semantics to all ERDF rules. This is because ERDF rules are derivation and not implication rules. Derivation rules are often identified with implications. For nonmonotonic rules (e.g. with negation-as-failure), this is no longer the case.

To define the intended (stable) models of an ERDF ontology, we need first to define grounding of ERDF rules.

Definition 19 (Grounding of an ERDF program). Let $V$ be a vocabulary and $r$ be an ERDF rule. We denote by $[r]_{V}$ the set of rules that result from $r$ if we replace each variable $x \in F \operatorname{Var}(r)$ by $v(x)$, for all mappings $v: F \operatorname{Var}(r) \rightarrow V$. Let $P$ be an ERDF program. We define $[P]_{V}=\bigcup_{r \in P}[r]_{V}$.

Below, we define the stable models of an ERDF ontology based on the coherent stable models of partial logic [7] (which, on extended logic programs, are equivalent [7] to Answer Sets [5]).

Definition 20 (Stable model). Let $O=\langle G, P\rangle$ be an ERDF ontology and let $M \in \mathcal{I}^{H}(O)$. We say that $M$ is a stable model of $O$ iff there is a chain of Herbrand interpretations of $O, I_{0} \leq \ldots \leq I_{k}$ such that $I_{k-1}=I_{k}=M$ and:

1. $I_{0} \in \operatorname{minimal}\left(\left\{I \in \mathcal{I}^{H}(O) \mid I \models \operatorname{sk}(G)\right\}\right)$.

2. For $0<\alpha \leq k$ :

$I_{\alpha} \in \operatorname{minimal}\left\{I \in \mathcal{I}^{H}(O) \mid I \geq I_{\alpha-1}\right.$ and $I \models \operatorname{Concl}(r)$, for all $r \in$ $\left.P_{\left[I_{\alpha-1}, M\right]}\right\}$, where

$P_{\left[I_{\alpha-1}, M\right]}=\left\{r \in[P]_{V_{O}}|I| \operatorname{Cond}(r), \forall I \in\left[I_{\alpha-1}, M\right]_{O}\right\}$.

The set of stable models of $O$ is denoted by $\mathcal{M}^{\text {st }}(O)$.

The following proposition shows that a stable model of an ERDF ontology $O$ is a Herbrand model of $O$.

Proposition 6. Let $O=\langle G, P\rangle$ be an ERDF ontology and let $M \in \mathcal{M}^{s t}(O)$. It holds $M \in \mathcal{M}^{H}(O)$.

On the other hand, if all properties are total, a Herbrand model $M$ of an ERDF ontology $O=\langle G, P\rangle$ is a stable model of $O$. This is because, in this case $M \in \operatorname{minimal}\left(\left\{I \in \mathcal{I}^{H}(O) \mid I \models \operatorname{sk}(G)\right\}\right)$ and $M \in \operatorname{minimal}\left\{I \in \mathcal{I}^{H}(O) \mid I \geq\right.$ $M$ and $I \models \operatorname{Concl}(r)$, for all $\left.r \in P_{[M, M]}\right\}$.

Proposition 7. Let $O=\langle G, P\rangle$ be an ERDF ontology, such that rdf s:subclass (rdf:Property, erdf:TotalProperty $) \in G$. Then, $\mathcal{M}^{s t}(O)=\mathcal{M}^{H}(O)$. 
From Proposition 2, it follows that if $r d f s: s u b c l a s s(r d f:$ Property, erdf:TotalProperty) $\in G$ then each $M \in \mathcal{M}^{H}(O)$ is a classical ERDF interpretation. Therefore, the above proposition shows that classical (boolean) Herbrand model reasoning on ERDF ontologies is a special case of stable model reasoning.

Similarly to [587], stable models do not preserve Herbrand model satisfiability. For example, let $O=\langle\emptyset, P\rangle$, where $P=\{p(s, o) \leftarrow \sim p(s, o)\}$, and $p, s, o \in U R I$. Then, $\mathcal{M}^{s t}(O)=\emptyset$, whereas there is a Herbrand model of $O$ that satisfies $p(s, o)$.

Definition 21 (Stable model entailment). Let $O=\langle G, P\rangle$ be an ERDF ontology and let $F$ be an ERDF formula. We say that $O$ entails $F$ under the (ERDF) stable model semantics, denoted by $O \models{ }^{s t} F$ iff for all $M \in \mathcal{M}^{s t}(O)$, $M \models F$.

For example, let $O=\langle\emptyset, P\rangle$, where $P=\{p(s, o) \leftarrow \sim q(s, o)\}$ and $p, q, s, o \in U R I$. Then, $O \models{ }^{s t} \sim q(s, o) \wedge p(s, o)$. Let $O=\langle G, P\rangle$, where

$G=\{r d f s: s u b c l a s s(r d f:$ Property, erdf:TotalProperty $)\}$ and $P$ is as in the previous example. Then, $O \models{ }^{s t} q(s, o) \vee p(s, o)$, but $O \not^{s t} \sim q(s, o)$ and $O \not{ }^{s t} p(s, o)$. This is the desirable result, since $q$ is a total property, and thus in contrast to the previous example, an open-world assumption is made for $q$. As another example, let $p, s, o \in U R I$, let $G=\{p(s, o)\}$, and let $P=\{\neg p(? x, ? y) \leftarrow$ $\sim p(? x, ? y)\}$. Then, $\langle G, P\rangle \models{ }^{s t} \sim p(o, s) \wedge \neg p(o, s)$ (note that $P$ contains a CWA on $p)$. Let $G=\{r d f: t y p e(p$, erdf:TotalProperty $), p(s, o)\}$ and let $P$ be as in the previous example. Then, $\langle G, P\rangle \models{ }^{s t} \forall ? x \forall ? y \quad(p(? x, ? y) \vee \neg p(? x, ? y))$ (see Proposition 1), but $\langle G, P\rangle \not \forall^{s t} \sim p(o, s)$ and $\left.\langle G, P\rangle \not\right|^{s t} \neg p(o, s)$. Indeed, the CWA in $P$ does not affect the semantics of $p$, since $p$ is a total property.

Let us now see a more involved example5. Consider the following ERDF program $P$, specifying some rules for concluding that a country is not a member state of the European Union (EU).

$\left(r_{1}\right) \quad \neg r d f:$ type $(? x$, EUMember $) \leftarrow r d f:$ type $(? x$, AmericanCountry $)$.

$\left(r_{2}\right) \quad \neg$ rdf: type $(? x$, EUMember $) \leftarrow$ rdf: type $(? x$, EuropeanCountry $)$, $\sim r d f:$ type $(? x$, EUMember $)$.

A rather incomplete ERDF ontology $O=\langle G, P\rangle$ is obtained by including the following information in the ERDF graph $G$ :

$\neg$ rdf: type(Russia, EUMember). rdf:type (Austria, EUMember). rdf:type(?x, EuropeanCountry).
rdf:type(Canada, AmericanCountry). rdf: type(Italy, EuropeanCountry). $\neg$ rdf:type(?x, EUMember $)$.

Using stable model entailment on $O$, it can be concluded that Austria is a member of EU, that Russia and Canada are not members of EU, and that it exists a European Country which is not a member of EU. However, it is also concluded that Italy is not a member of EU, which is a wrong statement. This is because $G$ does not contain complete information of the European countries

\footnotetext{
${ }^{5}$ For simplicity, the example namespace $e x$ : is ignored.
} 
that are EU members (e.g., it does not contain rdf:type(Italy, EUMember)). Thus, incorrect information is obtained by the closed-world assumption expressed in rule $r_{2}$. In the case that rdf:type(EUMember,erdf:TotalClass) is added to $G$ (that is, an open-world assumption is made for the class EUMember) then $\sim r d f$ :type(Italy, EUMember) and thus, $\neg$ rdf:type(Italy, EUMember) are not longer entailed. This is because, there is a stable model of the extended $O$ that satisfies $r d f$ :type (Italy, EUMember). Moreover, if complete information for all European countries that are members of EU is included in $G$ then the stable model conclusions of $O$ will also be correct (the closed-world assumption will be correctly applied). Note that, in this case $G$ will include $r d f$ :type(Italy, EUMember).

The following proposition shows that stable model entailment extends RDFS entailment from RDF graphs to ERDF ontologies.

Proposition 8. Let $G, G^{\prime}$ be RDF graphs such that $V_{G} \cap \mathcal{V}_{E R D F}=\emptyset, V_{G^{\prime}} \cap$ $\mathcal{V}_{E R D F}=\emptyset$, and $V_{G^{\prime}} \cap s k_{G}(\operatorname{Var}(G))=\emptyset$. It holds: $G \models^{R D F S} G^{\prime}$ iff $<G, \emptyset>\models^{s t}$ $G^{\prime}$.

Below we define the stable answers of a query $F$ w.r.t. an ERDF ontology.

Definition 22 (Stable answers). Let $O=\langle G, P\rangle$ be an ERDF ontology. A query $F$ is an ERDF formula. The (ERDF) stable answers of $F$ w.r.t. $O$ are defined as follows: $\operatorname{Ans}_{O}^{s t}(F)=\left\{v: F \operatorname{Var}(F) \rightarrow V_{O} \mid \forall M \in \mathcal{M}^{s t}(O): M \models\right.$ $v(F)\}$, where $v(F)$ is the formula $F$ after replacing all the free variables $x$ in $F$ by $v(x)$.

An ERDF ontology $O=\langle G, P\rangle$ is called simple if each rule in $P$ has the form $L_{0} \leftarrow L_{1}, \ldots, L_{k}, \sim L_{k+1}, \ldots, \sim L_{n}$, where each $L_{i}$ is an ERDF triple (positive or negative). The following proposition shows that the stable answers of a query $F$ w.r.t. a simple ERDF ontology can be computed through Answer Set Programming [5] on an extended logic program (ELP).

Proposition 9. Let $O=\langle G, P\rangle$ be a simple ERDF ontology and let $F$ be an ERDF formula. We can define an extended logic program $\Pi_{O}$ and a corresponding formula $F^{\prime}$ such that: The answers of $F^{\prime}$ according to the answer set semantics [5] of $\Pi_{O}$ coincide with $\operatorname{Ans}_{O}^{s t}(F)$.

Intuitively, $\Pi_{O}$ is generated as follows: (i) each $[\sim \mid \neg] p(s, o) \in L\left(V_{O} \mid\{\sim, \neg\}\right)$ is represented by $[\sim \mid \neg] \operatorname{Holds}(s, p, o)$, where Holds is a conventional predicate name and $p$ becomes a term, (ii) $s k(G)$ is represented as a set of facts, and (iii) semantics implicit in the definition of an ERDF interpretation is represented as rules. $\Pi_{O}$ is the union of the rules generated in (ii-iii).

\section{Related Work}

In this section, we briefly review extensions of web ontology languages with rules.

TRIPLE [15] is a rule language for the Semantic Web supporting RDF and a subset of OWL Lite [13]. It is based on F-Logic [11]. Part of the semantics of the $\mathrm{RDF}(\mathrm{S})$ vocabulary is represented in the form of pre-defined rules and not 
as semantic conditions on interpretations. TRIPLE includes a form of negationas-failure under the well-founded semantics [4. Strong negation is not used.

Flora-2 [20] is a rule-based object-oriented knowledge base system for reasoning with semantic information on the Web. It is based on F-logic [11] and supports metaprogramming, nonmonotonic multiple inheritance, logical database updates, encapsulation, dynamic modules, and two kinds of weak negation (specifically, Prolog negation and well-founded negation [4]). In Flora-2, anonymous resources are handled through skolemization (similarly to our theory).

Notation 3 (N3) provides a more human readable syntax for RDF and also extends RDF by adding numerous pre-defined constructs ("built-ins") for being able to express rules conveniently (see [17]). Remarkably, N3 contains a builtin (log:definitiveDocument) for making restricted completeness asumptions and another built-in (log:notIncludes) for expressing simple negation-as-failure tests. The addition of these constructs was motivated by use cases. However, N3 does not have any direct formal semantics for these constructs, and does not provide strong negation. In an extended version of this paper we will show how these N3 constructs can be mapped to ERDF.

OWL-DL [13] is an ontology representation language for the Semantic Web, that is a syntactic variant of the $\mathcal{S H O I N}(\mathbf{D})$ description logic and a decidable fragment of first-order logic. However, the need for extending the expressive power of OWL-DL with rules has initiated several studies, including the SWRL (Semantic Web Rule Language) proposal [10]. In [9], it is shown that this extension is in general undecidable. For an overview of (decidable) approaches of combining Description Logics with rules, see [3]. In several of these approaches, entailment on the extended with rules DL is based on first-order logic, that is both the DL component and the logic program are viewed as a set of first-order logic statements. Thus, negation-as-failure, closed-world-assumptions, and nonmonotonic reasoning cannot be supported. In contrast in our work, we support both weak and strong negation, and allow closed-world and open-world reasoning on a selective basis.

\section{Conclusions}

In this paper, we extended RDF graphs to ERDF graphs by allowing negative triples, and then to ERDF ontologies with the inclusion of derivation rules, allowing freely appearance of (meta)properties and (meta)classes in the body and head of the rules, all logical factors $\sim, \neg, \forall, \exists, \supset, \wedge, \vee$ in the body of the rules, and strong negation $\neg$ in the head of the rules. Moreover, the $\operatorname{RDF}(\mathrm{S})$ vocabulary was extended with the terms erdf:TotalProperty and erdf:TotalClass. We have developed the model-theoretic semantics of ERDF ontologies, called ERDF stable model semantics, showing that stable model entailment extends RDFS entailment on RDF graphs. We have shown that classical (boolean) Herbrand model reasoning is a special case of our semantics, when all properties are total. In this case, similarly to classical logic, an open-world assumption is made for all properties and classes. Allowing totalness of properties and classes to 
be declared on a selective basis and the explicit representation of closed-world assumptions (as derivation rules) enables the combination of open-world and closed-world reasoning in the same framework. For simple ERDF ontologies, our semantics can be computed through Answer Set Programming [5]. Future work concerns the support of datatype maps, including $X S D$ datatypes, and the extension of the ERDF vocabulary to other useful ontological categories possibly in accordance with [16].

\section{References}

1. A. Analyti, G. Antoniou, C. V. Damasio, and G. Wagner. Negation and Negative Information in the W3C Resource Description Framework. Annals of Mathematics, Computing $\&$ Teleinformatics (AMCT), 1(2):25-34, 2004.

2. Tim Berners-Lee. Design issues - architectual and philosophical points. Personal notes, 1998. Available at http://www.w3.org/DesignIssues/.

3. E. Franconi and S. Tessaris. Rules and Queries with Ontologies: A Unified Logical Framework. In Second International Workshop on Principles and Practice of Semantic Web Reasoning (PPSWR 2004), pages 50-60, 2004.

4. A. Van Gelder, K. A. Ross, and J. S. Schlipf. The well-founded semantics for general logic programs. Journal of the ACM, 38(3):620-650, 1991.

5. M. Gelfond and V. Lifschitz. Logic programs with classical negation. In Warren and Szeredi, editors, 7th International Conference on Logic Programming, pages 579-597. MIT Press, 1990.

6. Patrick Hayes. RDF Semantics. W3C Recommendation, 10 February 2004. Available at http://www.w3.org/TR/2004/REC-rdf-mt-20040210/.

7. H. Herre, J. Jaspars, and G. Wagner. Partial Logics with Two Kinds of Negation as a Foundation of Knowledge-Based Reasoning. In D.M. Gabbay and H. Wansing, editors, What Is Negation? Kluwer Academic Publishers, 1999.

8. H. Herre and G. Wagner. Stable Models are Generated by a Stable Chain. Journal of Logic Programming, 30(2):165-177, 1997.

9. I. Horrocks and P. F. Patel-Schneider. A Proposal for an OWL Rules Language. In 13th International Conference on World Wide Web (WWW'04), pages 723-731. ACM Press, 2004.

10. I. Horrocks, P. F. Patel-Schneider, H. Boley, S. Tabet, B. Grosof, and M. Dean. SWRL: A semantic web rule language combining OWL and RuleML. W3C Member Submission, 21 May 2004. Available at http://www . w3. org/Submission/2004/SUBM-SWRL-20040521/.

11. M. Kifer, G. Lausen, and J. Wu. Logical Foundations of Object-Oriented and Frame-Based Languages. Journal of the ACM, 42(4):741-843, 1995.

12. G. Klyne and J. J. Carroll. Resource Description Framework (RDF): Concepts and Abstract Syntax. W3C Recommendation, 10 February 2004. Available at http://www.w3.org/TR/2004/REC-rdf-concepts-20040210/.

13. D. L. McGuinness and F. van Harmelen. OWL Web Ontology Language Overview. W3C Recommendation, 10 February 2004. Available at http://www.w3.org/TR/2004/REC-owl-features-20040210/.

14. The rule markup initiative (ruleml). Available at http://www.ruleml.org.

15. M. Sintek and S. Decker. TRIPLE - A Query, Inference, and Transformation Language for the Semantic Web. In First International Semantic Web Conference on The Semantic Web (ISWC2002), pages 364-378. Springer-Verlag, 2002. 
16. H. J. ter Horst. Extending the RDFS Entailment Lemma. In 3rd International Semantic Web Conference (ISWC2004), pages 77-91, 2004.

17. Tim-Berners-Lee. Notation 3 - An RDF language for the Semantic Web. W3C Recommendation, 1998. Available at http://www .w3.org/DesignIssues/Notation3.html.

18. G. Wagner. A Database Needs Two Kinds of Negation. In 3rd Symposium on Mathematical Fundamentals of Database and Knowledge Base Systems (MFDBS'91), pages 357-371. Springer-Verlag, 1991.

19. G. Wagner. Web Rules Need Two Kinds of Negation. In 1st International Workshop on Principles and Practice of Semantic Web Reasoning (PPSWR'03), pages 33-50. Springer-Verlag, December 2003.

20. Guizhen Yang and Michael Kifer. Inheritance and Rules in Object-Oriented Semantic Web Languages. In 2nd International Workshop on Rules and Rule Markup Languages for the Semantic Web (RULEML'03), pages 95-110, 2003. 